\title{
Effets de l'incorporation de la biomasse d'azolla (Azolla pinnata) séchée dans les rations du poulet en aviculture traditionnelle améliorée
}

\author{
Bansé OUEDRAOGO ${ }^{*}$, Zara S. NIKIEMA ${ }^{2}$, Jean Sibiri ZOUNDI ${ }^{3}$ et Laya \\ SAWADOGO ${ }^{4}$ \\ ${ }^{1}$ Département de Production Animales / Institut de l'Environnement et de Recherches Agricoles (INERA), 04 \\ BP 8645 Ouagadougou 04, Burkina Faso. \\ ${ }^{2}$ Département de Production Végétale / Institut de l'Environnement et de Recherches Agricoles (INERA), 04 \\ BP 8645 Ouagadougou 04, Burkina Faso. \\ ${ }^{3}$ Département de Gestion des Ressources Naturelles et Systèmes de Production (GRN/SP)/Institut de \\ l'Environnement et de Recherches Agricoles (INERA), 04 BP 8645 Ouagadougou 04, Burkina Faso. \\ ${ }^{4}$ Université de Ouagadougou, UFR/SVT, 01 BP 7029 Ouagadougou 01Burkina Faso. \\ *Auteur correspondant; E-mail : banse_ouedraogo@yahoo.fr; Tel. (00 226) 78780061 /
}

(00 226) 70376152

Received: 12-10-2020

Accepted: 26-02-2021

Published: 28-02-2021

\section{RÉSUMÉ}

L'objectif de ce travail est de rechercher une voie alternative d'amélioration de l'alimentation et de la productivité avicole au Burkina Faso. L'étude vise à évaluer les effets de l'incorporation de la farine d'Azolla pinnata dans la ration alimentaire sur les performances de croissance des poulets de chair. Pour ce faire, une expérience a été conduite pendant 56 jours avec 120 poussins de chair âgés de 21 jours afin d'évaluer l'effet de l'incorporation de la farine d'Azolla pinnata comme ingrédient alimentaire dans la ration de poulets de chair. Le soja torréfié utilisé comme témoin a été substitué partiellement par la farine d'A.pinnata à des taux de 0,3 et 6\% correspondant à trois traitements alimentaires R0, R1, R2. A la fin de l'essai, une augmentation du poids des sujets avec les apports croissants d'A. pinnata dans les rations (R1 et R2) a été observée même si elle n'a pas été significative. Les indices de consommation n'étaient pas significativement différents. L'incorporation n'a engendré aucun effet néfaste sur le rendement carcasse des sujets nourris à base de la farine d'Azolla en comparaison aux sujets témoins. La substitution partielle du soja torréfié par la farine d'Azolla aux taux de 3\% et $6 \%$ a entraîné une diminution des coûts alimentaires par $\mathrm{kg}$ de poids vif de poulet.

(C) 2021 International Formulae Group. All rights reserved.

Mots clés : Aviculture traditionnelle améliorée, Azolla pinnata, poussins hybrides, performance de croissance.

\section{Effects of incorporating dried azolla (Azolla pinnata) biomass in chicken rations in improved traditional poultry farming}

\begin{abstract}
The objective of this work is to look for an alternative way to improve poultry feed and productivity in Burkina Faso. The study aims to evaluate the effects of the incorporation of Azolla pinnata meal in the feed ration on the growth performance of broilers. To this end, a 56-day experiment was conducted with 120 21-day-old broiler chicks to evaluate the effect of incorporating Azolla pinnata meal as a feed ingredient in the broiler ration.
\end{abstract}


The roasted soybean used as a control was partially substituted with A. pinnata meal at levels of 0,3 and $6 \%$ corresponding to three feed treatments R0, R1, R2. At the end of the trial, an increase in the weight of the subjects with increasing $A$. pinnata intakes in the rations (R1 and R2) was observed, although it was not significant. The consumption indices were not significantly different. Incorporation had no adverse effect on carcass yield in subjects fed Azolla meal compared to control subjects. Partial substitution of roasted soybean with Azolla meal at $3 \%$ and $6 \%$ resulted in lower feed costs per $\mathrm{kg}$ live weight of chicken.

(C) 2021 International Formulae Group. All rights reserved.

Keywords: Improved traditional poultry farming, Azolla pinnata, hybrid chicks, growth performance.

\section{INTRODUCTION}

$\mathrm{Au}$ Burkina Faso (BF) l'élevage constitue l'un des principaux piliers de l'économie nationale. Il est pratiqué par plus de $80 \%$ des ménages qui en tirent tout ou partie de leurs revenus. Il représente le deuxième secteur productif et constitue la première source de revenus monétaires des ménages ruraux (MRAH, 2013). Dans cet élevage, l'aviculture constitue une stratégie de survie pour les populations. En effet, l'aviculture traditionnelle est facilement accessible aux ménages ruraux et parvient donc à générer plus de revenu en raison du faible niveau requis en termes d'investissement initial (Grégoire et al. 2019). Il s'avère donc nécessaire et indispensable d'améliorer puis de promouvoir la production avicole traditionnelle améliorée. Mais cette aviculture est confrontée à plusieurs contraintes parmi lesquelles figure l'alimentation qui représente 60 à $80 \%$ du coût de production en aviculture (MARA, 2010). Les ingrédients conventionnels riches en protéines et en énergie deviennent non seulement rares mais également coûteux. L'utilisation des aliments locaux non conventionnels dans l'alimentation animale apparaît comme une solution endogène (Dahouda et al., 2013). A cet effet La recherche et la valorisation de ressources alimentaires alternatives disponibles localement devraient permettre d'améliorer la productivité des volailles tout en maîtrisant les coûts des intrants (Ouédraogo et al., 2015). Parmi ces ressources alimentaires alternatives, on $\mathrm{y}$ trouve l'Azolla, aliment d'origine végétale, très peu ou pas exploité pour l'alimentation animale qui n'entre pas en concurrence avec l'alimentation humaine (Dahouda et al., 2009).
Il s'agit d'aliments de substitution ou de remplacement des aliments conventionnels (Aboh et al., 2011). D'importants résultats en matière d'alimentation de la volaille avec la farine d'Azolla pinnata ont été obtenus par différentes études (Hêdji et al., 2014). En effet, Azolla peut être un supplément de protéines précieuses pour de nombreuses espèces animales comme les ruminants, la volaille, les porcs et les poissons (Hasan et al., 2009).

L'Azolla contient également en moyenne $5 \mathrm{~g}$ lysine $/ 100 \mathrm{~g}$ protéines et a un profil en acide aminé relativement bien équilibré (Leterme et al., 2009).

La bio-composition d'Azolla en fait l'un des substituts alimentaires les plus économiques, efficaces et durables pour la volaille. L'incorporation d'Azolla comme ingrédient protéique alternatif dans la ration de volaille pourrait donc rendre la production de volaille économique.

\section{MATERIEL ET METHODES Matériel \\ Période d'étude}

L'étude s'est déroulée dans un poulailler situé à l'intérieur de la station de recherche de Farakoba à Bobo Dioulasso Région Ouest du Burkina Faso du 15 avril 2019 au 15 juin 2019 soit une durée globale de deux (2) mois. L'étude a débuté au début de la période pluvieuse.

\section{Animaux d'expérience}

Le cheptel expérimental était composé de 120 poussins Isa Brown obtenus par incubation dans une couveuse électrique de capacité $36 \times 14$. Après éclosion, les poussins sont nourris pendant trois semaines (21 jours) avec des aliments poussins commercial. Ils 
sont ensuite répartis selon un dispositif aléatoire complètement randomisé en trois(3) lots de poids sensiblement égal et attribués de façon aléatoire aux régimes expérimentaux. Les 3 régimes utilisés dans l'essai sont théoriquement isoénergétiques et isoprotéiques.

\section{Ingrédients utilisés}

La production de l'Azolla pinnata a été réalisée grâce à quatre petits bassins de dimensions $2 \mathrm{~m} / 1 \mathrm{~m}$ situés à l'intérieur de la station de recherche. La récolte est faite quand l'A. pinnata a couvert toute la surface de l'eau. Une fois récoltée, l'A. pinnata est débarrassée de ses impuretés, puis séchée au soleil pendant 3 à 4 jours le temps nécessaire pour qu'elle sèche bien puis elle est réduite en farine grâce au moulin (Figure 1).

\section{Méthodologie}

Avant le début de l'essai, les poussins ont été élevés dans une poussinière durant trois (3) semaines puis transférés dans le bâtiment de croissance de la ferme qui est divisé en six compartiments (Tableau 1).

Les poulets sont élevés sur un sol en béton recouvert d'une litière de sciure de bois de $5 \mathrm{~cm}$ de hauteur dans les mêmes conditions d'humidité, de ventilation et de température. L'intérieur du bâtiment est cloisonné en six (06) boxes (de dimension $2 \mathrm{~m} / 1 \mathrm{~m}$ ) grâce à du grillage permettant une franche aération. Les mesures de prophylaxie sanitaire telles que l'hygiène, le vide sanitaire, et la prophylaxie médicale, ont été respectées pour éviter et prévenir l'apparition d'éventuelles pathologies.

\section{Prophylaxie médicale}

Le protocole de prophylaxie médicale appliqué est celui en vigueur dans le centre de recherche de Faraoba (Bobo Dioulasso) et reporté dans le Tableau 2. Les premières semaines, les poussins ont reçu un anti- stress, puis vaccinés contre la maladie de Newcastle et traités contre la coccidiose et les parasitoses gastro-intestinales (Tableau 2).

\section{Identification, mise en lot et transition alimentaire}

La répartition des poussins a été réalisée à l'âge de 21 jours. Le dispositif expérimental mis en place comportait trois traitements alimentaires (R0, R1 et R2) avec deux (2) répétions ( $\mathrm{A}$ et $\mathrm{B}$ ) par traitement. Chaque traitement alimentaire a été affecté à un lot de 40 sujets répartis en deux sous lots de 20 sujets. La mise en lot a été faite de telle sorte qu'il n'y ait pas de différence significative entre les poids moyens des sujets des différents lots. Les sujets de chaque lot ont été identifiés grâce à des bagues en plastique placées sur l'aile droite.

Après la mise en lot, les poussins ont été soumis à une transition alimentaire d'une semaine pendant laquelle l'aliment commercial a été progressivement remplacé par les aliments expérimentaux pour amener les oiseaux à s'habituer progressivement à la ration expérimentale. L'eau de robinet leur a été donnée à volonté et renouvelée chaque jour.

\section{Rations alimentaires}

$\mathrm{La}$ formulation des rations expérimentales a été faite sur la base des résultats d'analyse bromatologique au laboratoire du centre de recherche de certaines matières premières (Farine d'Azolla, soja torréfiée) et des valeurs bromatologiques rapportées par Ayssiwede et al. (2011) pour les autres ingrédients.

Les trois rations (R0, R1 et $\mathrm{R} 2$ ) ont été formulées et utilisées pour la croissancefinition. La ration R0 a servi de témoin et correspond à $0 \%$ d'incorporation de l'A. pinnata. Dans les rations expérimentales R1 et R2 le soja torréfié a été partiellement substitué par la farine d'A. pinnata aux taux respectifs de $3 \%$ et $6 \%$ (Tableau 3 ).

\section{Collecte de données}

Les poids des oiseaux sont notés de façon hebdomadaire et les quantités d'aliments consommés (servis et refus) sont relevées par pesée quotidienne puis les carcasses pesées à l'abattage. Ce processus nous permet de calculer des paramètres comme (1) la consommation alimentaire, (2) la Croissance pondérale, (3) l'Indice de Consommation (IC), (4) le taux de mortalité, (5) le rendement Carcasse. 


\section{Analyse statistique des données}

Les données ont été saisies à l'aide du tableur Microsoft Office Excel. 2010. Ce même outil a été utilisé pour la construction des tableaux et des graphiques. Les analyses statistiques ont été effectuées à l'aide du logiciel R 3.5.1. Les effets des aliments sur la consommation alimentaire, le gain de poids, l'indice de consommation et le rendement carcasse ont été testés par analyse de la variance (ANOVA) utilisant le model de Tukey HSD au seuil de 0,05 pour la séparation des moyennes des différents paramètres étudiés.

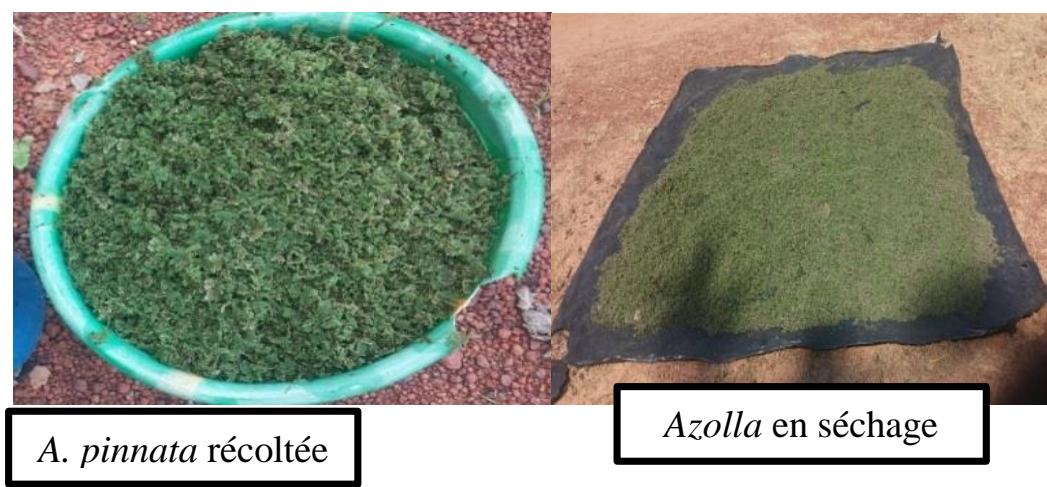

Figure 1 : Production et séchage de l'Azolla (Ouédraogo, 2019).

Tableau 1 : Schéma du dispositif expérimental.

\begin{tabular}{ccccccc}
\hline blocs & \multicolumn{3}{c}{ Répétition 1 } & \multicolumn{3}{c}{ Répétition 2 } \\
\hline Traitements & R0 & R1 & R2 & R0 & R1 & R2 \\
\hline Nombre de sujet & 21 & 21 & 21 & 21 & 21 & 21 \\
\hline
\end{tabular}

Tableau 2 : Prophylaxie médicale appliquée.

\begin{tabular}{lll}
\hline Age (jour) & Actes & Produits utilisés \\
\hline 1 & $\begin{array}{l}\text { Vaccination contre les maladies de } \\
\text { Newcastle }\end{array}$ & HB1 (Trempage de bec, boisson) \\
\hline $1,2,3,4$ & $\begin{array}{l}\text { Administration antistress et } \\
\text { antibiotique }\end{array}$ & COLITERRAVET \\
\hline 9 & $\begin{array}{l}\text { Vaccination contre la maladie de } \\
\text { Gumboro }\end{array}$ & $\begin{array}{l}\text { Hypragumboro-CH80 (trempage et eau de } \\
\text { boisson) }\end{array}$ \\
\hline $9,10,11$ & Administration anti-stress & COLITETRAVET \\
\hline $17,18,19,20$ & Administration d'anticoccidiens & Amprolium 20\% \\
\hline 21 & $\begin{array}{l}\text { Rappel vaccination contre la maladie } \\
\text { de Gumboro et Newcastle }\end{array}$ & $\begin{array}{l}\text { Hypragumboro-CH8 (trempage et eau de } \\
\text { boisson) }\end{array}$ \\
\hline $21,22,23$ & Administration anti-stress & Néoxyvital \\
\hline 28,56 & $\begin{array}{l}\text { Administration d'antiparasitaires et } \\
\text { de vitamines }\end{array}$ & $\begin{array}{l}\text { Citrate de pipérazine, Albendazole plus } \\
\text { vitamines }\end{array}$ \\
\hline
\end{tabular}


Tableau 3 : Composition centésimale des différentes rations.

\begin{tabular}{lccc}
\hline Ingrédients & \multicolumn{3}{c}{ Rations } \\
\cline { 2 - 4 } & $\mathbf{R 0}$ & $\mathbf{R 1}$ & $\mathbf{R 2}$ \\
\hline Maïs & 52 & 52 & 52 \\
\hline Son de blé & 17,5 & 17,5 & 10 \\
\hline Soja torréfié & 16 & 13 & 6 \\
\hline Farine d'Azolla & 0 & 7,5 & 7,5 \\
\hline Tourteau d'arachide & 7,5 & 4 & 4 \\
\hline Farine de poisson & 4 & 2,5 & 2,5 \\
\hline CMV & 2,5 & 0,5 & 0,5 \\
\hline NaCl & 0,5 & 100 & 100 \\
\hline Total & 100 & & \\
\hline
\end{tabular}

\section{RESULTATS}

\section{Composition bromatologique des rations au} laboratoire

Le Tableau 4 montre que les teneurs en Matière Azotée Totale (MAT) et en énergie métabolisable (EM) des trois rations (R0, R1 et $\mathrm{R} 2)$ sont presque identiques. Le traitement témoin (R2) (6\% d'Azolla pinnata) est plus riche en matière grasse (MG) que les traitements R1 (3\% d'A. pinnata) et R0 (0\% d'A. pinnata). Les traitements R1 et R2 ont une teneur plus élevée en cellulose brute (CB) que la ration T0 (Tableaux 4).

\section{Effet de l'incorporation d'Azolla sur la consommation alimentaire}

Les consommations alimentaires obtenues chez les sujets des différents traitements alimentaires sont consignées dans le (Tableau 5).

De la première ( $\left.1^{\text {ère }}\right)$ à la huitième $\left(8^{\text {ème }}\right)$ semaine d'expérience, la consommation alimentaire moyenne des différents lots n'a pas connu de différence significative entre les traitements au seuil de 5\%. En effet, sur l'ensemble de la période expérimentale (S1S8), l'analyse statistique révèle que l'aliment R2 est le mieux consommé avec $66,79 \pm 1,80 \mathrm{~g}$ par poulet/ jour. La différence entre ces valeurs de la consommation est statistiquement non significative $(\mathrm{P}=0,655)$ au seuil de $5 \%$. L'aliment R2 et l'aliment R1 $(65,21 \pm 1,81 \mathrm{~g})$ sont les mieux consommés que R0 $(64,83 \pm 2,08 \mathrm{~g}) . \mathrm{Au}$ total, l'apport en farine d'Azolla a entraîné une meilleure consommation des rations (Tableau 5).

\section{Effet sur la mortalité des poulets.}

Au départ, 120 poussins ont été utilisés pour l'expérimentation pour une durée de 56 jours. De faibles mortalités ont été enregistrées au cours de l'étude.

$\mathrm{Au}$ total, trois (03) mortalités ont été enregistrées. Ceci représente un taux de $(2,5 \%)$ du cheptel expérimental.

Ces mortalités ont été réparties comme suit :

(a).Deux (02) cas de mortalité observés au niveau du lot nourri avec la ration R1.

(b).Un (01) cas de mortalité enregistré au niveau du lot nourri avec la ration $\mathrm{R} 2$.

En revanche, aucun cas de mortalité n'a été enregistré au niveau du lot témoin (R0).

Toutes les mortalités sont survenues à la première semaine d'expérimentation chez les sujets des traitements R1 et R2. Le taux de mortalité globale de $(2,5 \%)$ observée pour l'ensemble des lots est suffisamment faible pour être attribué à l'effet de l'incorporation de la farine d'azolla dans la ration alimentaire. Des études plus poussées avec des taux d'incorporation plus élevés permettront de mieux éclairer ce constat, surtout que l'autopsie n'a pas révélé des lésions signes caractéristiques d'une maladie particulière. 


\section{Effet de l'incorporation de l'Azolla sur le poids vif des sujets}

L'effet de l'incorporation de l'Azolla dans les différentes rations sur l'évolution des poids vifs des animaux en fonction du temps est présenté sur la Figure 2.

Du démarrage jusqu'à la 4ème semaine d'âge (première semaine d'essai $=\mathrm{S} 1$ ), l'effet de l'incorporation de la farine d'azolla sur l'évolution pondérale des poussins a été significatif $(\mathrm{P}=0,002)$. Il en est de même à la semaine $\mathrm{S} 2 \quad(\mathrm{P}=0,044)$ et la semaine $\mathrm{S} 3$ $(\mathrm{P}=0,044)$. Sur toute a durée de l'essai ( $\mathrm{S} 1$ S8) le poids moyen des poulets a été de $1397,07 \pm 47,51 \mathrm{~g}$ pour les sujets du R2, $1307,73 \pm 50,54 \mathrm{~g}$ pour ceux du R1 et $1265,07 \pm 53,19 \mathrm{~g}$ pour les sujets du R0.

Sur les 8 semaines de, l'étude l'effet de l'incorporation de la farine d'azolla n'a pas été significatif $(\mathrm{P}=0,181)$ sur le poids des poulets.

Donc en tenant compte des différentes rations, on constate qu'il n'y a pas une différence significative au seuil de $5 \%$. Les poulets de R2 (6\%) ont enregistré un poids moyen plus élevé (1397 g) suivi de ceux du R1 (1307 g) et enfin ceux du R0 (1265 g).

A la fin de l'essai, une augmentation du poids des sujets avec les apports croissants de la farine d'A. pinnata dans les rations expérimentales a été observée même si elle n'a pas été significative $(\mathrm{P}<0,05)$ (Figure 2$)$.

\section{Effets sur le Gain Moyen Quotidien (GMQ)}

Les GMQ des sujets des différents traitements (R0, R1,R2) sont restés quasisimilaires, c'est-à-dire, sans différence significative durant la période d'étude (S1-
$\mathrm{S} 8)(\mathrm{P}=0,188)$ même si $\mathrm{R} 2$ et $\mathrm{R} 1$ ont présenté légèrement des meilleurs GMQ que le témoin R0 (Tableau 6)

\section{Effet sur l'Indice de Consommation (IC) alimentaire}

Les valeurs de l'indice de consommation des sujets soumis aux différents traitements sont présentées dans le tableau 6 . Aucune différence significative n'a été observée entre les valeurs calculées sur les quatre périodes de l'expérimentation. Sur l'ensemble de la durée de l'expérimentation (S1-S8), l'IC des poulets nourris au traitement témoin R0 était en moyenne de 3,87 $\pm 1,33$ contre $3,75 \pm 1,44$ pour $\mathrm{R} 1$ et $3,76 \pm 1,71$ pour R2. Il n'y a pas eu de différence statistiquement significative entre les traitements $(\mathrm{P}=0,981)$. (Tableau 7)

\section{Effet sur les caractéristiques de la carcasse}

Le Tableau 8 donne les résultats de l'évaluation du rendement carcasse des poulets en fonction des traitements.

Les rendements carcasses ont varié entre $74,46 \%$ et $76,66 \%$. Les rendements carcasses moyens observés ont été de $74.46 \%$, $76.66 \%$ et $76.30 \%$ respectivement pour les oiseaux nourris aux traitements R0, R1 et R2. L'incorporation n'a engendré aucun effet néfaste sur le rendement carcasse des sujets nourris à base de la farine de farine d'Azolla en comparaison aux sujets témoins. En général, ce sont les poulets ayant consommé les régimes $\mathrm{R} 2$ et celui à R1 de farine d'Azolla qui présentent les meilleurs rendements. (Tableau 8)

Tableau 4 : Composition bromatologique des rations en $\%$.

\begin{tabular}{lccc}
\hline \hline \multirow{2}{*}{ Nutriments } & \multicolumn{3}{c}{ Traitements } \\
\cline { 2 - 4 } & $\mathbf{R 0}$ & $\mathbf{R 1}$ & $\mathbf{R 2}$ \\
\hline MS $(\%)$ & 91,42 & 91,48 & 91,59 \\
\hline MAT $(\%)$ & 20,50 & 20,65 & 20,92 \\
\hline MG $(\%)$ & 4,02 & 4,10 & 4,74 \\
\hline CB $(\%)$ & 3,70 & 4,20 & 4,25 \\
\hline EM $(\mathrm{kcal})$ & 2913 & 2906 & 2909 \\
\hline \hline
\end{tabular}

MS : Matière Sèche ; MAT : Matière Azotée Totale ; MG : Matière Grasse ; CB : Cellulose Brute ; EM : Énergie Métabolisable. 
Tableau 5 : Consommation alimentaire des sujets.

\begin{tabular}{cccccc}
\hline \multicolumn{7}{c}{ Consommation alimentaire } \\
\hline Période & $\mathbf{R 0}(\mathbf{0 \%})$ & $\mathbf{R 1}(\mathbf{3 \% )}$ & $\mathbf{R 2}(\mathbf{6 \% )}$ & $\mathbf{P r}(>\mathbf{F})$ & Signification \\
\hline S1-S2 & $56,75 \pm 3,36$ & $50,71 \pm 1,85$ & $50,9 \pm 1,85$ & 0,154 & NS \\
\hline S3-S4 & $62,49 \pm 5,56$ & $60,80 \pm 3,44$ & $67,24 \pm 2,06$ & 0,496 & NS \\
\hline S5-S6 & $67,42 \pm 2,94$ & $71,46 \pm 2,01$ & $69,4 \pm 1,55$ & 0,463 & NS \\
\hline S7-S8 & $72,66 \pm 1,69 \mathrm{a}$ & $77,87 \pm 2,02 \mathrm{ab}$ & $79,65 \pm 2,35 \mathrm{~b}$ & 0,053 & NS \\
\hline S1-S8 & $64,83 \pm 2,08$ & $65,21 \pm 1,81$ & $66,79 \pm 1,80$ & 0,655 & NS \\
\hline
\end{tabular}

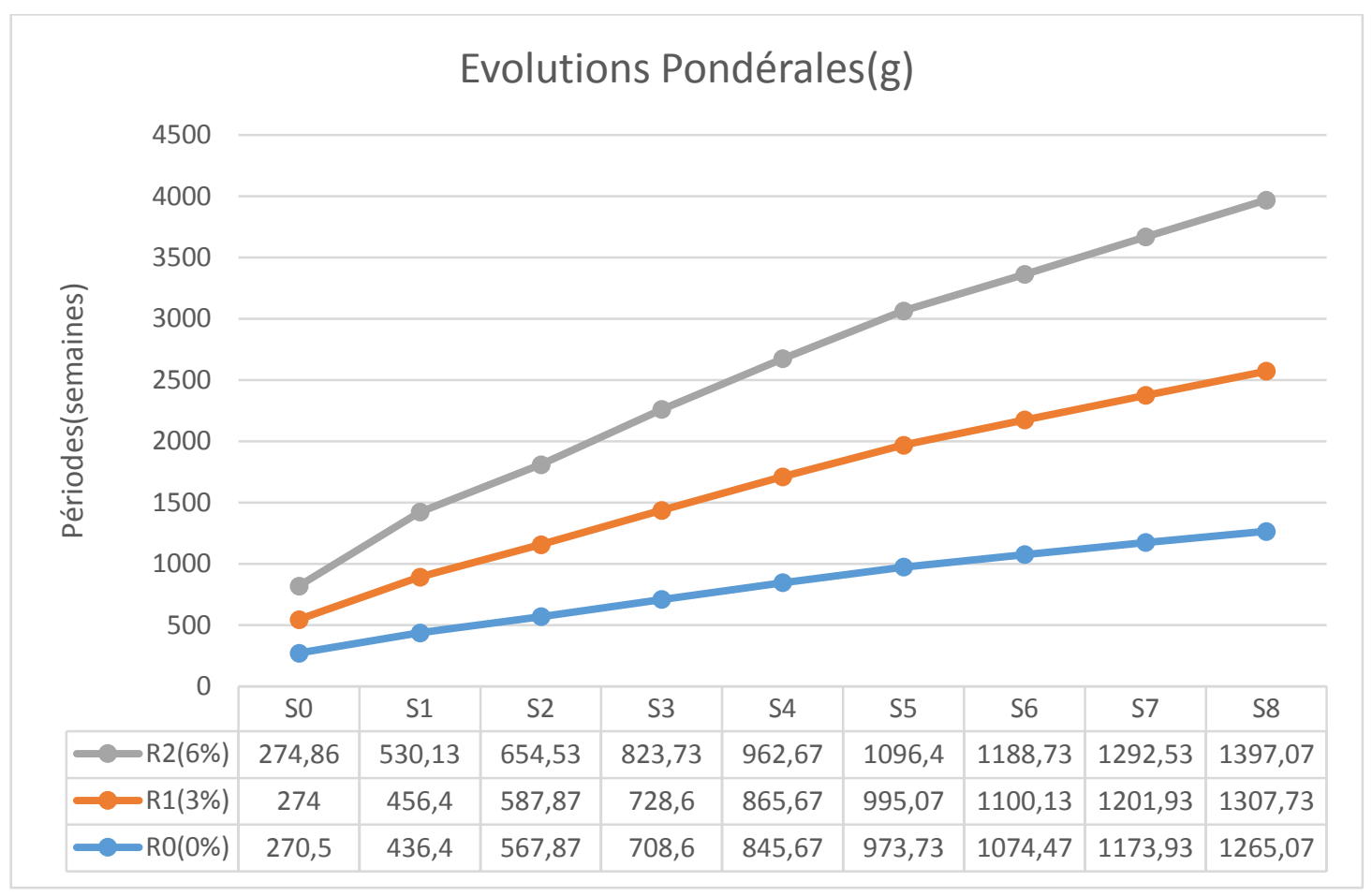

Figure 2 : Courbe d'évolution pondérale des sujets de chaque lot.

Tableau 6 : Gains Moyens Quotidiens des sujets.

\begin{tabular}{cccccc}
\hline \multicolumn{7}{c}{ Gains Moyens Quotidiens } \\
\hline Période & R0 $(\mathbf{0 \%})$ & R1 $(\mathbf{3 \%})$ & $\mathbf{R 2 ~}(\mathbf{6 \% )}$ & $\operatorname{Pr}(>\mathbf{F})$ & Signification \\
\hline S1-S2 & $21,24 \pm 1,58$ & $22,42 \pm 1,67$ & $27,12 \pm 2,21$ & 0,063 & NS \\
\hline S3-S4 & $19,84 \pm 1,00$ & $19,84 \pm 1,00$ & $22,01 \pm 1,04$ & 0,225 & NS \\
\hline S5-S6 & $16,34 \pm 1,45$ & $16,75 \pm 1,45$ & $16,15 \pm 1,38$ & 0,955 & NS \\
\hline S7-S8 & $13,61 \pm 2,67$ & $14,83 \pm 2,26$ & $14,88 \pm 1,64$ & 0,902 & NS \\
\hline S1-S8 & $17,76 \pm 0,92$ & $18,46 \pm 0,86$ & $20,04 \pm 0,92$ & 0,188 & NS \\
\hline
\end{tabular}


Tableau 7 : Indices de consommation des sujets.

\begin{tabular}{cccccc}
\hline \multicolumn{5}{c}{ Indices de consommation } \\
\hline Période & $\mathbf{R}(\mathbf{0 \%})$ & $\mathbf{R}(\mathbf{3} \%)$ & $\mathbf{R}(\mathbf{6 \% )}$ & $\operatorname{Pr}(>\mathbf{F})$ & Signification \\
\hline S1-S2 & $2,76 \pm 0,89$ & $2,34 \pm 0,65$ & $2,17 \pm 1,14$ & 0,848 & NS \\
\hline S3-S4 & $3,14 \pm 0,60$ & $3,06 \pm 0,25$ & $3,08 \pm 0,41$ & 0,990 & NS \\
\hline S5-S6 & $4,23 \pm 0,99$ & $4,34 \pm 0,86$ & $4,44 \pm 1,00$ & 0,984 & NS \\
\hline S7-S8 & $5,35 \pm 0,25$ & $5,25 \pm 0,23$ & $5,35 \pm 0,33$ & 0,944 & NS \\
\hline S1-S8 & $3,87 \pm 1,33$ & $3,75 \pm 1,44$ & $3,76 \pm 1,71$ & 0,981 & NS \\
\hline
\end{tabular}

Tableau 8 : Rendements carcasse des poules obtenus avec les différents lots.

\begin{tabular}{c|ccc}
\hline \multirow{2}{*}{ Lot } & \multicolumn{3}{|c}{ Paramètres } \\
\cline { 2 - 4 } R0 & Poids Vif & Poids de la carcasse & Rendement carcasse \\
\hline R1 & 1265 & 942 & 74,46 \\
R2 & 1307 & 1002 & 76,66 \\
\hline Signification & 1397 & 1066 & 76,30 \\
\hline
\end{tabular}

\section{DISCUSSION}

\section{Composition bromatologique des rations}

Les compositions bromatologiques des trois (3) rations formulées dans le cadre de cette étude sont conformes aux recommandations de l'ITAVI (2003). Les teneurs en énergie et en matières azotées des rations R1 et R2 ont été comparables que celles de la ration $\mathrm{R} 0$ à base de farine de soja torréfié uniquement, témoignant ainsi que l'Azolla pinnata est riche en ces éléments nutritifs (Alalade et Iyayi, 2006). Le même constat est fait par Paudel et al. (2015). Par ailleurs, l'augmentation des taux de fibres des rations expérimentales (R1 et R2) à cause de l'A. pinnata corrobore les travaux de Mamata et al. (2018), ainsi que celui d'Anitha et al. (2016) qui avaient rapporté que l'A. pinnata a un taux élevé de fibre. Cela est susceptible d'augmenter significativement le taux de fibre des aliments si elle est incorporée à un taux élevé.

Effet de l'Azolla sur la croissance des poulets

Nos résultats ont montré que la substitution partielle du soja torréfiée par la farine d'Azolla pinnata n'a pas d'effet significatif sur l'évolution pondérale et les Gains Moyens Quotidiens des poulets. Ce résultat corrobore celui d'Alalade et Iyayi (2006) au Nigéria qui avaient montré que la farine d 'A. pinnata peut être incorporée jusqu'à $10 \%$ dans la ration alimentaire des poussins sans affecter négativement la croissance de ces derniers. Des résultats meilleurs avaient été obtenus par Basak et al. (2002) au Bengladesh qui a constaté à partir de la sixième $\left(6^{\mathrm{e}}\right)$ semaine d'âge, que les poulets de chair nourris avec une ration incorporant $5 \% \mathrm{~d}$ 'A. pinnata en substitution au sésame avaient des poids 
corporels significativement améliorés par rapport au témoin ( $0 \%$ d'A. pinnata).

\section{Consommation alimentaire}

Les différents taux de substitution partielle de soja torréfié par la farine d'Azolla pinnata ont induit des effets non significatifs sur la consommation alimentaire individuelle des animaux pendant toute la période expérimentale sans pour autant affecter l'indice de consommation. Notre étude a révélé aussi que plus l'aliment contient de l'Azolla mieux il est consommé. Ce résultat est contraire à celui de Mangesh et al. (2018) en Inde qui ont constaté une diminution de la consommation alimentaire des poulets de chair avec 2,5\%,5\% d'inclusion de l'A. pinnata par rapport au témoin ( $0 \%$ d'inclusion de l'A. pinnata). Ceci est en accord avec les résultats obtenus par Alalade et al. (2006) qui ont enregistré des diminutions de l'ingestion d'aliments jusqu'à 5\% mais augmentation de la consommation alimentaire lors de l'incorporation d'Azolla au taux de 7,5\% dans l'alimentation des poussins de chair.

\section{Indice de conversion alimentaire (IC)}

Les indices de consommation obtenus dans la cadre de cette étude $(3,75$ à 3,87$)$ sont supérieurs à l'indice de consommation de référence des poulets de chair à croissance rapide qui est de (1,8 à 2,2). Cependant, ils sont meilleurs à ceux des poulets locaux rapportés par Ouattara (2008) au Burkina Faso $(4,4$ à 4,9) et par Bello (2010) au Sénégal (5,8 à 7,7). Les indices de consommation des lots nourris avec les rations incorporant l'A. pinnata en substitution partielle au soja torréfié n'ont pas été significativement différents de celle du lot témoin. Cela montre une bonne aptitude des poulets locaux à valoriser les rations incorporant l'A. pinnata Dans le même ordre d'idée que nos travaux, Basak et al. (2002) ont constaté pour l'indice de consommation des poulets de chair un effet non significatif avec $5 \%$ d'incorporation de l'A. pinnata en substitution au sésame au cours de la période de 2 à 4 semaines et une amélioration significative au cours de la période de 5 à 7 semaines d'âge.

\section{Caractéristiques des carcasses}

Notre étude a montré que la substitution partielle du soja torréfié par la farine d'Azolla pinnata n'a pas eu d'effet significatif sur les rendements carcasses des poulets. Ce résultat est en accord avec celui de Bhattacharyya et al. (2014) qui ont trouvé non significatives les caractéristiques de la carcasse des poulets de chair avec 4,5 et 5,5\% d'azolla dans la ration. Ce résultat corrobore également celui de Basak et al. (2002) au Bengladesh qui avaient constaté que la substitution du sésame par l'A. pinnata aux taux de 5\%,10\% et $15 \%$ n'entrânait pas de différence significative sur les rendements carcasse des poulets de chair.

\section{Évaluation de la rentabilité}

Les prix des ingrédients sont ceux appliqués sur le marché lors de leur acquisition pour la conduite de l'essai, excepté celui du kilogramme de la farine d'Azolla $p$. qui a été estimé à 100 FCFA. Nous constatons que la substitution partielle du soja torréfié par la farine d'Azolla a entrainé une réduction du prix des rations R1 (3\% d'A. pinnata) et R2 (6\% $\mathrm{d}^{\prime} A$. pinnata $)$ par rapport à la ration $\mathrm{R} 0(0 \%$ d'A.pinnata). Cela est dû au fait que le prix de l'A. pinnata a été faible par rapport au prix du soja (350 FCFA/kg) sur le marché.

La substitution partielle du soja torréfié par la farine d'Azolla aux taux de 3\% (ration $\mathrm{R} 1$ ) et $6 \%$ (ration R2) a entrainé une diminution des coûts alimentaires par $\mathrm{kg}$ de poids vif de poulet (C. kg PV). Ceci corrobore les résultats de Basak et al. (2002) qui ont obtenu un coût du kilogramme $(\mathrm{kg})$ de poulet de chair significativement $(\mathrm{P}<0,05)$ meilleur dans l'alimentation avec 5\% de farine d'azolla. L'incorporation de l'Azolla en substitution partielle au soja torréfié constitue donc une alternative intéressante pour assainir l'environnement et diminuer les charges liées à l'alimentation des poulets qui peuvent représenter $60 \%$ à $80 \%$ des charges des exploitations avicoles dans un système d'intégration agriculture - élevage comme indiqué par Hêdji et al. (2015). Nos résultats corroborent ceux de Deepesh et al. (2016) qui constataient que l'incorporation de $5 \%$ et $7,5 \%$ de l'A. pinnata en substitution au sésame 
permet de diminuer les coûts de production du poulet de chair.

\section{Mortalité}

La présente étude a montré que l'A. pinnata n'a pas eu d'effet négatif sur l'état sanitaire des poulets. Ceci est en accord avec Basak et al. (2002) ainsi que Paudel et al. (2015) qui avaient expérimenté l'A. pinnata sur des poulets de chair à des taux d'incorporation relativement élevés et avaient montré que l'A. pinnata n'a pas d'effet négatif sur l'état sanitaire des poulets. Ces pertes sont minimes et n'affectent pas la pertinence des analyses statistiques. Aucune maladie ou lésion organique n'a été relevée à l'autopsie.

\section{Conclusion}

Pour pallier le problème lié au cout élevé de l'aliment, plusieurs études ont porté sur l'incorporation de ressources non conventionnelles végétales locales dans la ration du poulet de chair. L'analyse chimique a montré qu'Azolla pinnata est riche en protéines. L'étude a montré que les rations ayant reçu une incorporation d'Azolla étaient meilleures que les témoins teoins en termes de performances de croissance. Les poids vifs des poulets de chair ont été plus élevés dans les rations ayant reçu un taux d'incorporation de $3 \%$ et $6 \%$ d'Azolla par rapport au témoin. Les performances pondérales, le niveau d'ingestion alimentaire et l'indice de consommation des poussins de chair observés ont montré que l'inclusion de $6 \%$ d'Azolla pinnata est optimale pour une substitution partielle de la farine de soja torréfiée dans la ration d'alimentation de poulets de chair. La disponibilité de cette ressource et la facilité de sa culture en bassins en font une alternative pour la réduction du coût de production des poulets de chair en système d'élevage traditionnel amélioré. Cette forme de valorisation de cette plante aquatique envahissante peut contribuer à réduire son impact environnemental.

\section{CONFLIT D'INTÉRÊTS}

Les auteurs déclarent qu'ils n'ont pas de conflit d'intérêts.

\section{CONTRIBUTIONS DES AUTEURS}

$\mathrm{OB}$ et ZSN ont contribué à l'élaboration et l'édition du protocole de recherche. OB a assuré la rédaction du manuscrit et a effectué la collecte, la saisie et l'analyse des données sous la supervision de ZSJ et LS.

\section{REMERCIEMENTS}

Les auteurs adressent leurs sincères remerciements à Monsieur Ouédraogo Mahamadī, étudiant à l'Université Polytechnique de Bobo Dioulasso pour son soutien pendant le suivi de l'essai. Ils remerciements également Ouédraogo Souleymane, chercheur à l'Institut Environnemental et de Recherches Agricoles(INERA) de Farakoba pour ses contributions fortement appréciées pour a production de 1 'azolla.

\section{REFERENCES}

André BA, Serge EPM, Théodora GSA, Guy AM. 2011. Performance pondérale et caractéristiques des carcasses des poulets de chairalimentés avec des rations alimentaires à base de graines de Mucuna pruriens. International Journal of Biological and Chemical Sciences, 5(6): 2306-2316 DOI: http://dx.doi.org/10.4314/ijbcs.v5i6.11

Alalade OA, Iyayi EE. 2006. Chemical composition and the feeding value of Azolla (Azolla pinnata) meal for egg-type chicks. International Journal of Poultry Science, $\quad \mathbf{5}(2)$ : 137-141. DOI: http://dx.doi.org/10.3923/ijps.2006.137.1 41

Anitha KC, Rajeshwari YB, Prasanna SB, Shilpa SJ. 2016. Nutritive évaluation of Azolla as livestock feed. Journal of Experimental Biology and Agricultural Sciences, 4(6): 670-674. DOI: http://dx.doi.org/10.18006/2016.4(Issue6 ). 670.674

Ayssiwede SB, Dieng A, Bello H, Chrysostome CAAM, Hane MB, Mankor A, Dahouda M, Houinato MR, Hornick J L, Missohou A. 2011. Effects of Moringa oleifera (Lam.) leaves meal incorporation 
in diets on growth performances, carcass characteristics and economics results of growing indigenous Senegal chickens. Pak. J. Nutr., 10(12): 1132-1145

Basak B, Ahsan HP, Muhammad SR, Sharif UT, Bimol CR. 2002. Azolla (Azolla pinnata) as a feed ingredient in broiler ration. International Journal of Poultry Science, 1(1): 29-34. DOI: http://dx.doi.org/10.3923/ijps.2002.29.34

Bello H. 2010. Essai d'incorporation de la farine de feuilles de Moringa oleifera dans l'alimentation chez les poulets indigènes du Sénégal : Effets sur les performances de croissance, les caractéristiques de la carcasse et le résultat économique. Thèse de Doctorat d'état en médecine vétérinaire, École Inter-États des Sciences et Médecine Vétérinaires (E.I.S.M.V.), Université Check Anta Diop, Dakar-Sénégal, 84p.

Bhattacharyya A, Shukla PK, Roy D, Shukla M. 2014. Effect of feeding Azolla (Azolla pinnata) at different levels on carcass quality characteristics and cut up parts of commercial broilers. Proceeding of VI conference of Indian Meat Science A association and National symposium on "Sustainable Meat Production for Nutritional Security and Consumer Wellbeing: Challenges and Strategies". DUVASU, Mathura, India, p. 42.

Dahouda M, Toléba SS, Senou M, Youssao AKI, Hambuckers A, Hornick JL. 2009. Les ressources alimentaires nonconventionnelles utilisables pour la production aviaire en Afrique: valeurs nutritionnelles et contraintes. Ann. Méd. Vét., 153: 5-21. DOI: http://hdl.handle.net/2268/90677

Dahouda M, Adjolohoun S, Senou M, Toleba SS, Abou M, Vidjannagni DS, Kpodékon M, Youssao AKI. 2013. Effets des aliments contenant des folioles de Moringa oleifera Lam et des aliments commerciaux sur les performances de croissance des lapins (Oryctolagus cuniculus). International Journal of Biological and Chemical Sciences, 7(5): 1838-1852. DOI: 10.4314/ijbcs.v7i5.5
Deepesh BM, Debashis R, Vinod K, Amitav B, Muneendra K, Raju K, Shalini V. 2016. Effect of feeding azolla (Azolla pinnata) meal on the performance, nutrient utilization and carcass characteristics of Chabro chicken. Indian Journal of Poultry Science, 51(3): 259-263. DOI: http://dx.doi.org/10.5958/09748180.2016 .00060

Grégoire N, Walter O, Ayao M, Simplice BA, 2019. Analyse de l'importance socioéconomique de l'aviculture familiale dans le Département de Salemata au Sénégal. International Journal of Biological Chemical Sciences, 13(7): 3131-3143. DOI: 10.4314/ijbcs.v13i7.13

Hasan MR, Chakrabarti R. 2009. Use of algae and aquatic macrophytes as feed in smallscale aquaculture: a review. FAO Fisheries and Aquaculture Technical Paper. No. 531, Rome, 123p.

Hêdji CC, Marcel H, Frédéric H, Emile F. 2015. Assainissement de l'environnement par la valorisation des ressources non conventionnelles en alimentation de porcs en croissance. International Journal of Biological Chemical Sciences, 9(4): 1929-1936. DOI: http://dx.doi.org/10.4314/ijbcs.v9i4.18

Hêdji CC, Diane NS, Kpoguè G, Marcel RH, Emile DF. 2014. Valorisation de Azolla spp, Moringa oleifera, son de riz, et de coproduits de volaille et de poisson en alimentation animale: synthèse bibliographique. Journal of Applied Biosciences, 81: 7277-7289. DOI: http://dx.doi.org/10.4314/jab.v81i1.4

Institut Technique de l'Aviculture (ITAVI). 2003. Le marché mondial des viandes de volailles: la production de poulets de chair en climat chaud. ITAVI, Rennes, $110 \mathrm{p}$.

Leterme P, Londono AM, Munoz JE, Suarez J, Bedoya CA, Souffrant WB, Buldgen A. 2009. Nutritional value of aquatic ferns (Azolla filiculoides Lam. and Salvinia molesta Mitchell) in pigs. Animal Feed Science and Technology, 149: 135-148. DOI: 
http://dx.doi.org/10.1016/j.anifeedsci.20 09.10 .002

Mamata J, Abdul A, Anadamoy M, Shiv MS, Siddhnath, Satyanarayana B, Bhogeshwa C, Bhagchand C. 2018. Effect of Azolla (Azolla pinnata) feed on the growth of broiler chicken. Journal of Entomology and Zoology Studies, 6(3): 391-393. https://www.entomoljournal.com/archive s/2018/vol6issue3/PartF/6-2-252-137.

Mangesh K, Rajesh KD, Dinesh J, Rajesh N, Tribhuwan S, Umesh KP, Subhash K, Siyag SS. 2018. Effect of Inclusion of Sun Dried Azolla (Azolla pinnata) at Different Levels on the Growth and Performance of Broiler Chicks. Journal of Animal Research, 8(4): 629-632. DOI: http://dx.doi.org/10.30954/2277940X.08.2018.13

Ministère des Ressources Animales et Halieutiques(MRAH). 2013. Journée Nationale du Paysan 16ème Edition: Banfora les 25,26 et 27 AVRIL. MRAH, Burkina Faso. www.spcpsa.gov.bf/.../doc.../42document-mrah-jnp-16-2013-banfora

Ministère des Ressources Animales et Halieutiques (MRAH). 2010. Politique Nationale de Développement Durable de l'Elevage au Burkina Faso. MRAH,
Burkina Faso, 54p. http://www.mcdburkina.bf/documentatio $\mathrm{n} /$

Ouattara S. 2008. Utilisation des graines de Acacia macrostachya Reichend comme source de protéines dans l'alimentation des poulets de chair. Mémoire de Diplôme d'Etudes Approfondies(DEA) en Gestion Intégrée des Ressources Naturelles, Option : Nutrition et Alimentation Animale, Ingénieur de Développement Rural(IDR), Université Polytechnique de Bobo Dioulasso(UPB), Burkina Faso, 69 p.

Ouédraogo B, Balé B, Zoundi SJ, Sawadogo L. 2015. Caractéristiques de l'aviculture villageoise et influence des techniques d'amélioration sur ses performances zootechniques dans la province du Sourou, région Nord-Ouest Burkinabè. Int. J. Biol. Chem. Sci., 9(3): 1528-1543. DOI:

http://dx.doi.org/10.4314/ijbcs.v9i3.34

Paudel DR, Pramila D, Kailash PT, Dahal A. 2015. Azolla as an economic substitute to soybean based feed for poultry. Int. J. Appl. Sci. Biotechnol., 3(4): 619-625. DOI: http://dx.doi.org/10.3126/ijasbt.v3i4.136 36 